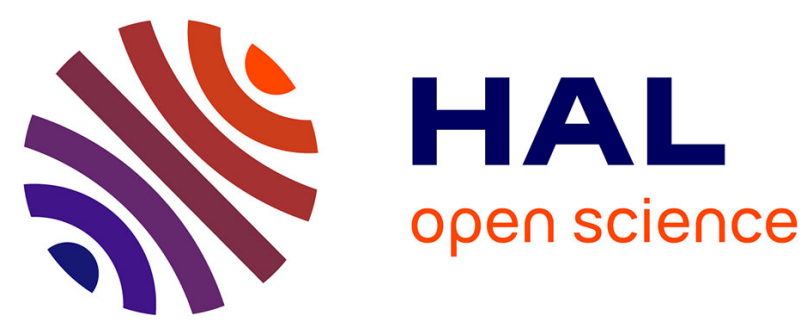

\title{
Industry IoT Gateway for Cloud Connectivity
}

Iveta Zolotová, Marek Bundzel, Tomáš Lojka

\section{To cite this version:}

Iveta Zolotová, Marek Bundzel, Tomáš Lojka. Industry IoT Gateway for Cloud Connectivity. IFIP International Conference on Advances in Production Management Systems (APMS), Sep 2015, Tokyo, Japan. pp.59-66, 10.1007/978-3-319-22759-7_7 . hal-01431176

\section{HAL Id: hal-01431176 \\ https://hal.inria.fr/hal-01431176}

Submitted on 10 Jan 2017

HAL is a multi-disciplinary open access archive for the deposit and dissemination of scientific research documents, whether they are published or not. The documents may come from teaching and research institutions in France or abroad, or from public or private research centers.
L'archive ouverte pluridisciplinaire HAL, est destinée au dépôt et à la diffusion de documents scientifiques de niveau recherche, publiés ou non, émanant des établissements d'enseignement et de recherche français ou étrangers, des laboratoires publics ou privés. 


\title{
Industry IoT Gateway for cloud connectivity
}

\author{
Iveta Zolotová, Marek Bundzel, Tomáš Lojka \\ Dept. of Cybernetics and Artificial Intelligence, FEI TU of Košice, Slovak Republic \\ \{iveta.zolotova, marek.bundzel, tomas.lojka\}@tuke.sk
}

\begin{abstract}
New approaches and technologies like Internet of Things (IoT), cloud computing and Big Data are giving rise to another industrial revolution. We propose here an implementation of an industrial gateway architecture adopting the idea of IoT, intelligent methods, Machine-to-Machine and CyberPhysical Systems. The proposed gateway creates a virtual representation of the physical world scanning the technological layer's devices in real time. It creates a uniform communication interface for the heterogeneous technological layer, enables self-management of devices, diagnostics and self-reconfiguration to improve Quality of Service aided with cloud SCADA and MES services. We have tested the proposed gateway in an experimental setup with a programmable logic controller.
\end{abstract}

Keywords: Cyber-Physical System, Gateway, Internet of Things.

\section{Introduction}

Industry is a heterogeneous system consisting of various heterogeneous subsystems [1]. The subsystems must be interconnected to reach the complexity needed for an optimal performance of a plant [2,3]. This need for interconnection leads to the next industrial evolution step defined as the Industry 4.0. The term "Industrie 4.0" (German for "industry") refers to the fourth industrial revolution. It originates from a project in the high-tech strategy of the German government, which promotes the computerization of manufacturing. [4,5]. The first industrial revolution increased production and decreased difficulty of manual work by using steam power. The second revolution did the same with the help of electric power and the third revolution brought electronics and IT to further automate production. The idea of the latest industrial revolution was presented at the Hanover Fair in 2011 [2].

Prof. Dr. Ing. Detlef Zühlke (Scientific Director of Innovative Factory Systems at the German Research Center for Artificial Intelligence) is sometimes called the "Father of Industry 4.0". The goal is to create more successful companies quickly capable to endure in the global competition [5].

Improvement of the interconnection of the industrial subsystems is related to the concept of the Internet of Things (IoT) [5], especially because of IoT aims to improve the interconnection of the digital and the physical world. Industry 4.0 in the contrary to the typical industry architecture significantly improves management of processes. IoT improves the processes of collecting, analyzing and processing the valuable and

adfa, p. 1, 2011.

(C) Springer-Verlag Berlin Heidelberg 2011 
now easily accessible information originated in every part of the plant. The industrial IoT changes the machine-machine and machine-human interactions, comprehensive interoperability and intelligence aids to improve analytical description of the environment and better decisions can be taken. [4], [6].

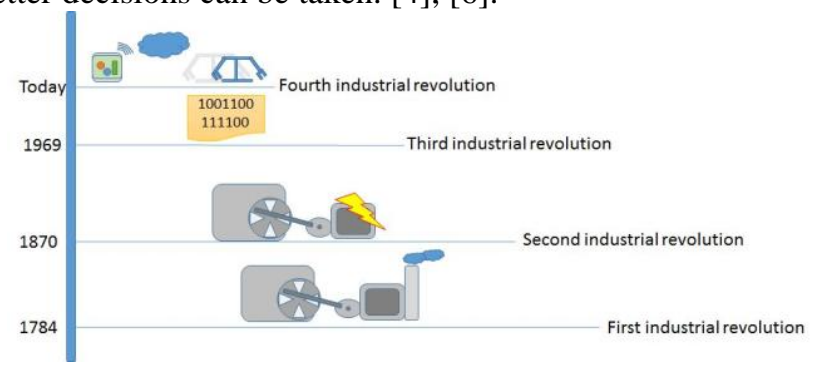

Fig. 1. Chronology of the industrial revolutions

Plants are comprised of different subsystems, modules, devices, machines operating with various communication protocols and interfaces. The problem is that the subsystems do not provide a unified connection to the technological layer and to the higher layer of the industrial hierarchical model. We propose implementation of the IoT in the Supervisory Control and Data Acquisition layer (SCADA). Typically the sensors of the technological layer produce large amounts of data; the SCADA layer collects it and provides the data to the Manufacturing Execution System (MES). This decreases interoperability between the technological layer and MES. The plants typically use strictly defined fixed interfaces for the communication within each layer between the layers of the hierarchy. This constraint does not allow ad-hoc behavior of the plant. Our goal is to create ad-hoc environment connecting technological layer with higher layers. The connections will be not constrained by fixed interfaces between the layers but automatically ad-hoc included and ad-hoc accessible for every layer.

\section{Industry 4.0 and IoT.}

Industry 4.0 brings greater flexibility, adaptability, self-organization, selfoptimization, self-diagnostics and leverages interaction between business and customer relationship, increases safety, reliability, autonomy and efficiency. The technological part is represented by a cyber-physical system [6]. Every product is individually described by the connection to business and customer layers. Integration of intelligent autonomy and monitoring with increasing automation into industry was important. It is represented by Cyber-Physical Systems (CPS) and IoT. [3].

Modern factories exceed the boundaries of the traditional ones. The factories are able to react to unpredicted situations autonomously and to select the optimal responses with regard to the plant or the market. The interconnection of the processes and plants is improved and the network security is better. The boundaries of the new industry are exceeded between the regions and between the different plants. 


\section{The Concept of Industrial Connection with IoT.}

Industry 4.0 connects machines, workers, and factory systems into a network. New technological approaches of IoT, cloud computing, M2M (machine-to-machine), CPS and Big data are implemented into the network. Each of the technologies increases productivity. [7, 8]. We focus on IoT facilitating the interconnection of heterogeneous factory environments. The communication within the factory and the digital model of the real factory states are improved. The proposed interconnection enables better analysis and decision making. The position of IoT in the hierarchical model is depicted in Fig. 2.

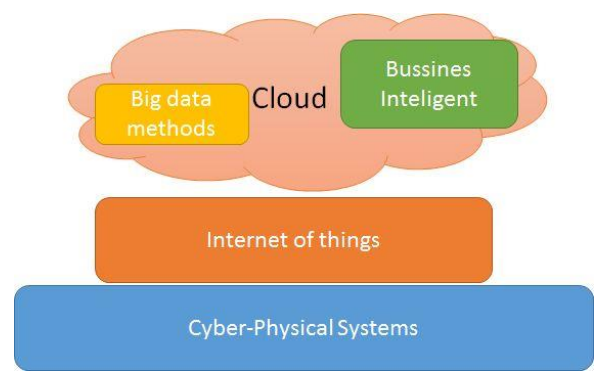

Fig. 2. Industrial connection with IoT, CPS, cloud, and Big data

IoT creates a virtual (digital) representation of the physical world. The software applications access the virtual representations of the devices only. This solution is more suited for the world representation management when using various physical devices $[9,10]$. This layer must implement ad-hoc behavior and it must be manageable by central or distributed nodes [11,12].

The IoT layer runs a software surveyor for detection and identification of new devices. It enables adaptation of any physical layer and its integration into the factory architecture. Data/information is accessible to every application inside the plant or inside/outside the plant respectively. The devices detected for the first time are looked up in a devices database and included into the device ontology. [4]. Measurement frequency, measurement range, device position etc. describe the device.

The connection to the technological layer is crucial and it influences the plant's Quality of Service (QoS) significantly. The technological layer of a heterogeneous system will not feature composite communication interfaces in Industry 4.0. The technological layer consists of CPS and smart products. These enable easier but not uniform connection to the technological layer. We propose a solution for the IoT connection to the technological layer here. We have designed a concept of IoT gateway that is described below. The proposed gateway supports communication with a cloud. SCADA and MES services may be implemented in the cloud as we have described in [13]. 


\section{Concept Definition of the IoT Gateway}

The important part of SCADA is the connection to the technological layer. This connection is crucial for data forwarding to the higher layers of the factory architecture. Therefore, we propose an IoT gateway connecting the physical devices and the higher layers of the Information and Control System (ICS). Regular and stochastic changes occur in a real word environment and the IoT gateway must adapt to the changes. The gateway's main role is the development of a virtual representation of the physical world enabling data collection and forwarding. The gateway's functionality must be robust to fulfill the requirements of all layers in the factory architecture.

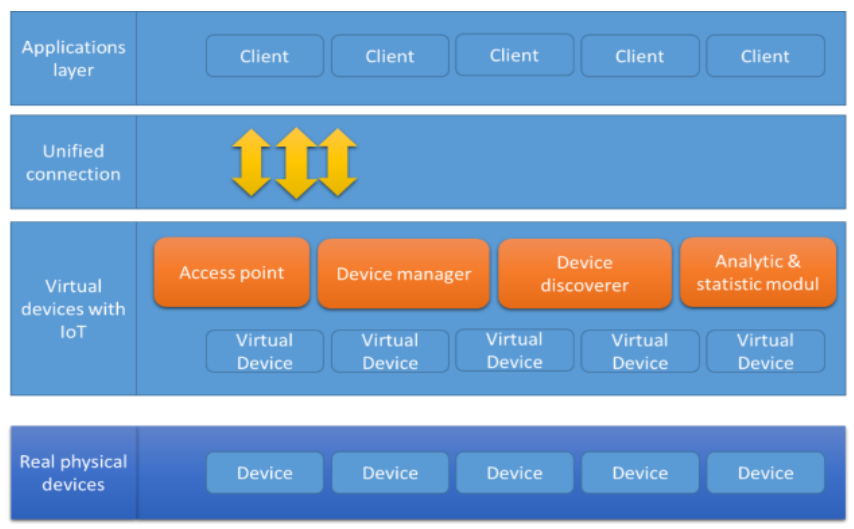

Fig. 3. Concept definition of IoT in the industry.

The IoT gateway distinguishes between the physical device and its virtual representation. That enables implementation of device management in the cases of failure or for saving energy when redundant measurements are taken. Additionally the virtual representation of the devices simplifies the connection between the cloud application and the physical devices (sensors/actuators). The gateway offers a unified connection to the virtual representations of the devices so that any application may use it.

The virtual representation of a device does not simply mirror the state of the device. Functionality for enhanced description of the device, its properties and measurements is added. $[9,10]$. The IoT gateway provides a complex representation of the instances.

We have defined the main tasks the IoT gateway will perform to reach the best QoS:

- Data forwarding - the gateway is responsible for data forwarding from the technological layer to the higher layers of the factory architecture. [11].

- Gateway management - the gateway is a key integration element in the entire factory architecture. A fixed and no-manageable gateway will cause fewer problems with adaptivity and implementation. [11]. 
- Device management - enables runtime configuration of devices, setting the statuses, functionality modes, errors acknowledgements and other. [11].

- Data analysis - the IoT gateway monitors and analyses data in real time using statistics and artificial intelligence. This module cooperates with the device management to maintain QoS, reliability and fault tolerant operation. The faults are detected and the faulty devices are substituted by the available redundant devices. Data analysis with the device management enable self-configuration, adaptive and robust behavior with regard to the technological layer connected to the IoT gateway. The goal is to achieve autonomous management of devices without the need of human intervention aided by M2M communication.

- Diagnostics - the IoT gateway detects errors and faults in the entire technological layer and in the IoT gateway itself.

The operation of the proposed IoT gateway is divided into the management and runtime parts.

\subsection{Management Part of the IoT Gateway}

Management part of the IoT gateway detects, identifies and networks new devices in real-time. The IoT gateway creates a virtual instance of a device after having detected it. A definition of the device is needed to create the virtual instance. The definition may contain measurement ranges, device's location and power management information, lists of fault states, error states and error messages. If the definition of the virtual instance is inferred from the physical device it is uploaded to the device database [10]. The device manager will then create the virtual instance and forward it to the runtime.

The virtual instance communicates with the device in the runtime. The data exchanges are based on events to reduce the communication load. However, the devices in Industry 4.0 should be manageable; at least the device's status must be settable.

This industrial solution enables to contact new devices. The contacted devices must provide metadata describing themselves to be correctly recognized. The IoT gateway creates the virtual instances based on the metadata. The virtual instances are frequently refreshed based on the corresponding events. The inactive physical devices are set to idle state by the device manager. Additional functionality is the management of the running virtual devices. This implies additional ability to update the physical and virtual instances in real time. The management part provides additional information to the data access point of the IoT gateway and the access point provides it to the higher layers (to MES, for example). The access point also collects information on configuration of virtual and physical instances from SCADA layer.

The diagnostics module enables management of the physical devices, the virtual instances and of the IoT gateway to ensure reliable control of the devices and data transfers from and to the technological layer. The diagnostics module also enables problem identification and invokes events describing the critical or the alarm states. 


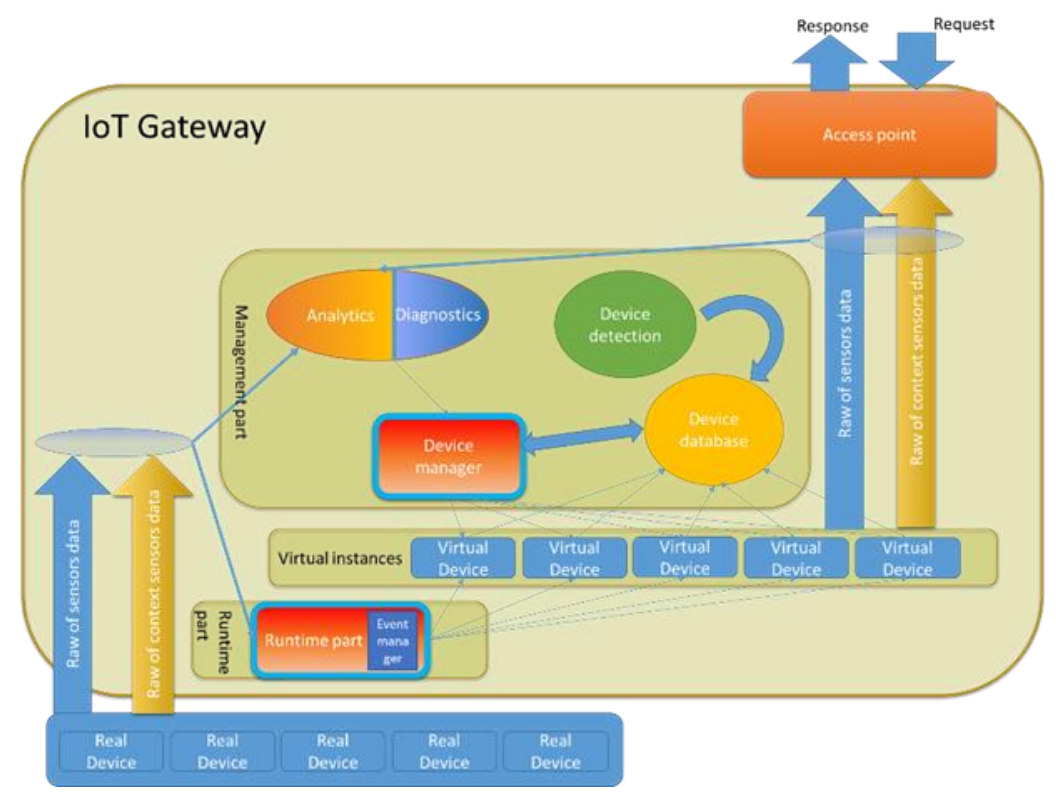

Fig. 4. Management and runtime parts of the IoT gateway

\subsection{The Runtime Part of the IoT Gateway}

Runtime part operates with the virtual instances created for the physical devices by the management part. Runtime part is responsible for finding new devices and updating the virtual instances. The virtual instances are easily accessible via the unified connection interface of the IoT gateway.

In the case of a fault occurring on the physical device the virtual instance may link itself to another redundant physical device or self-reconfigure. The functionality of the faulty device is substituted by functionality of another device. The runtime part runs an event manager that keeps the physical devices and the virtual instances synchronized.

\section{The Implemented Solution}

We have not implemented the full functionality of the above described concept so far. We are try to identify and to use progressive approaches to improve the interconnection of the heterogeneous technological level and the cloud SCADA and MES services. We have designed a clustering method for grouping redundant devices. Substitutes for the faulty devices are easy to find and robustness is increased.

We have developed the management and the runtime parts of the IoT gateway. We have implemented the software agent scanning the defined subnets and identifies all the connected devices. The implemented management part selects the devices with the known communication interfaces and stores them in the device database. After 
that virtual instances for the devices are created and a synchronization interval is set up.

We have used PLC CompactLogix L23E from Rockwell Automation in our experiment. The PLC has been detected and identified by IoT gateway. An industrial IoT gateway must communicate via various communication protocols. The proposed IoT gateway uses a wide scale of industrial communication protocols; CIP (Common Industrial Protocol) was used for communication with the PLC in our experiment.

We have developed a GUI showing the IoT gateway processes using WPF and MS Visual Studio 2013. A list of the available devices found by the IoT gateway is shown in Fig. 5. The IP address of the PLC is shown in the Device register tab. Its virtual instance properties are displayed in the Instance properties tab. The virtual instance was automatically created upon reception of the metadata downloaded from the PLC.

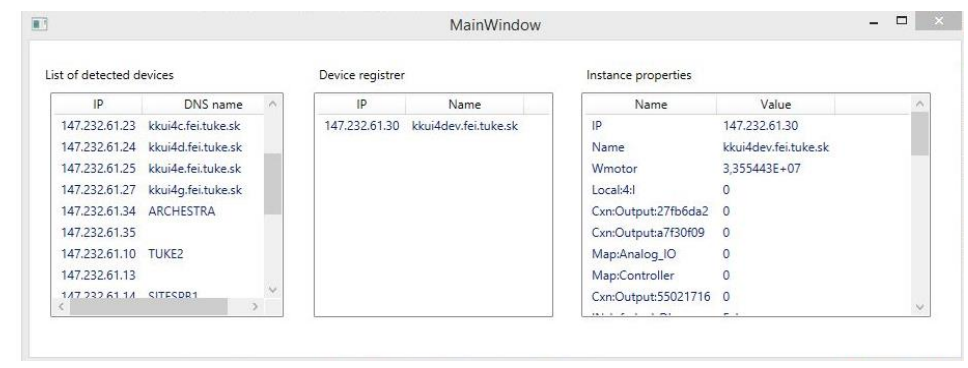

Fig. 5. Graphic representation IoT gateway processes

\section{Conclusions and Future Work}

New industrial revolution based on CPS, IoT, cloud computing, and Big Data is on the way. $[4,12]$. We have focused CPS and IoT for increased industrial autonomy, flexibility, efficiency, self-configuration, self-adaptiveness and robustness. Important part is communication in industry. Heterogeneity of the technological layer makes communication problematic. We have designed and implemented an IoT gateway creating a unified connection to the technological layer aided by IoT. This proposed gateway allows management of devices, identification of new devices, reading context data of the devices, self-reconfiguration, diagnostics and fault tolerance by creating virtual representations of the physical devices.

The future work includes implementation of artificial intelligence methods for classification of devices and their communication interfaces, for analysis of their behaviors and implementation of M2M to achieve self-managing behavior of the IoT gateways and their cooperation.

Acknowledgements. This publication is the result of the Project implementation: University Science Park TECHNICOM for Innovation Applications Supported by Knowledge Technology, ITMS: 26220220182, supported by the Research \& Devel- 
opment Operational Programme funded by the ERDF (50\%) and by grant KEGA 001 TUKE-4/2015 (50\%).

\section{References}

1. Atzori, L., Iera, A., Morabito, G.: The Internet of Things: A Survey. In: Computer Networks, Vol. 54, No. 15, pp. 2787-2805 (2010)

2. Li, J., Biennier, F., Ghedira Ch.: An Agile Governance Method for Multi-tier Industrial. In: Advances in Production Management Systems. Value Networks: Innovation, Technologies, and Management, pp.506-513, Springer Berlin Heidelberg (2012)

3. Breiner, K., Görlich, D., Maschino, O., Meixner, G., Zühlke, D: Run-time adaptation of a universal user interface for ambient intelligent production environments. In: HumanComputer Interaction. Interacting in Various Application Domains, pp. 663-672, Springer Berlin Heidelberg (2009)

4. Zuehlke, D.: SmartFactory-Towards a factory-of-things. In: Annual Reviews in Control, 34(1), pp. 129-138 (2010)

5. Bill Lydon: The 4th Industrial Revolution, Industry 4.0, Unfolding at Hannover Messe 2014, Automation.com (2014), http://www.automation.com/automation-news/article/the4th-industrial-revolution-industry-40-unfolding-at-hannover-messe-2014

6. Brizzi, P., Conzon, D., Khaleel, H., Tomasi, R., Pastrone, C., Spirito, A.M., Knechtel, M., Pramudianto, F., Cultrona, P.: Bringing the Internet of Things along the manufacturing line: A case study in controlling industrial robot and monitoring energy consumption remotely. In: Emerging Technologies \& Factory Automation (ETFA), 2013 IEEE 18th Conference, pp.1-8 (2013)

7. Spiess, P., Karnouskos, S., Guinard, D., Savio, D., Baecker, O., Souza, L. M. S. D., Trifa, V.: SOA-based integration of the internet of things in enterprise services. In: Web Services, 2009. ICWS 2009. IEEE International Conference, pp. 968-975, IEEE (2009)

8. Broy, M.: Cyber-physical systems: Innovation durch softwareintensive eingebettete Systeme. Springer, Berlin (2010)

9. Schuh, G., Potente, T., Thomas, C., \& Hauptvogel, A.: Cyber-Physical Production Management. In: Advances in Production Management Systems. Sustainable Production and Service Supply Chains, pp. 477-484, Springer Berlin Heidelberg (2013)

10. Jammes, F.; Smit, H.: Service-oriented paradigms in industrial automation. In: Industrial Informatics, IEEE Transactions, vol.1, no.1, pp.62-70 (2005)

11. Xiong, N., Svensson, P.: Multi-sensor management for information fusion: issues and approaches. Information fusion, 3(2), pp. 163-186 (2002)

12. Copie, A., Fortis, T., Munteanu, V.I., Negru, V.: From Cloud Governance to IoT Governance. In: Advanced Information Networking and Applications Workshops (WAINA), 2013 27th International Conference, pp.1229-1234 (2013)

13. Lojka,T., Zolotová, I.: Improvement of Human-Plant Interactivity via Industrial CloudBased Supervisory Control and Data Acquisition System. In: Advances in Production Management Systems (APMS) 2014. Vol. 440, no. Part 3 (2014), pp. 83-90.

14. Peniak,P.: Cloud Computing and integration of manufacturing information systems with process control, University of Žilina, FEE, DCIS - PIPA 5.2.14 Automation. 2014 